\title{
ESTUDO DAS LESÕES ORAIS EM PACIENTES PEDIÁTRICOS SUBMETIDOS À QUIMIOTERAPIA
}

\author{
Naiah Enéas da Silva Almeida'; Maria da Conceição Andrade ${ }^{2}$ \\ 1. Voluntária PEVIC, Graduanda em Medicina, Universidade Estadual de Feira de Santana, e-mail: nai_eneas@ hotmail.com \\ 2. Orientadora, Departamento de Saúde, Universidade Estadual de Feira de Santana, e-mail: conceicao.icb@gmail.com
}

PALAVRAS-CHAVE: Oncologia; Odontopediatria; Lesões orais.

\section{INTRODUÇÃO}

O câncer é uma patologia caracterizada pela proliferação descontrolada de células anormais em qualquer local do organismo. As neoplasias mais frequentes que acometem crianças e adolescentes são as leucemias, tumores do sistema nervoso central e linfomas (INCA, 2016). No Brasil, bem como nos países desenvolvidos, representa a primeira causa de morte por doença nessa faixa etária. No entanto, a evolução dos protocolos para tratamento das neoplasias pediátricas tem determinado um aumento progressivo nos índices de cura (INCA 2016). No entanto, a terapia antineoplásica traz diversas complicações, sendo as lesões orais uma das principais. Dados da literatura mostram que cerca de $40 \%$ dos pacientes oncológicos apresentam repercussões bucais agudas, como mucosite, aftas, candidíase, herpes, gengivites e hemorragias gengivais, infecções (Martins et al, 2002).

A mucosite é uma alteração da mucosa bucal, sendo uma complicação não hematológica resultante da terapia antineoplásica (Sasada et al, 2013). É a manifestação oral mais comum, ocorrendo entre 40 a $100 \%$ dos pacientes em uso de quimioterápicos e em $100 \%$ daqueles submetidos à radioterapia na região de cabeça e pescoço (Cunha, 2010). Pode agravar, acometendo todo o trato gastrointestinal.

O presente trabalho tem como objetivos analisar as lesões orais em crianças submetidas à quimioterapia, bem como fatores etiológicos relacionados ao câncer e o perfil sociodemográfico da população do estudo.

\section{MATERIAL E MÉTODOS OU METODOLOGIA}

Trata-se de estudo epidemiológico de corte transversal feito no Hospital Estadual da Criança (HEC), Feira de Santana - BA. A população estudada foram crianças do setor da Oncologia. Os critérios de inclusão foram: admissão na unidade entre setembro de 2016 a agosto de 2017, possuir diagnóstico de câncer, idade inferior a 18 anos, estar em tratamento ou já tê-lo concluído no HEC. Foram excluídos os pacientes com tumores benignos ou que abandonaram o tratamento. Foi aplicado questionário sobre características sociodemográficas e história médica-odontológica, bem como o Termo de Assentimento Livre e Esclarecido. Os dados foram descritos por meio de frequências absolutas e percentuais relativas. O programa utilizado para a compilação dos dados foi o Statistical Package for Social Science - SSPS e as informações foram sistematizadas em tabelas e gráficos com o auxílio do Excel.

\section{RESULTADOS}

Do total de 66 pacientes, 53\% são do sexo masculino e 47\%, do sexo masculino. A faixa etária mais acometida no sexo feminino foi de 6 a 12 anos (40\%), enquanto que no sexo masculino foi de 0 a 5 anos $(51,6 \%)$. A maioria dos pacientes de ambos os sexos, acima de $70 \%$, eram oriundos da zona urbana e viviam em moradias com 2 a 4 pessoas no total $(54,3 \%$ e $48,4 \%$ para os sexos feminino e masculino, respectivamente) e nenhum deles conviviam com mais de 8 pessoas na mesma residência. As genitoras apresentaram, de modo geral, melhor escolaridade em comparação aos genitores. No total, a maioria das mães referiram Ensino Médio Completo (27,3\%). Já a maioria dos genitores $(34,8 \%)$, referiu Ensino Fundamental Incompleto e nenhum apresentou Ensino Superior Completo. A maioria das 
crianças tinha entre 0 e 5 anos e a escolaridade mais relatada foi Ensino Fundamental Incompleto (42,9\% sexo feminino e $38,7 \%$ sexo masculino). A maioria das famílias incluídas na pesquisa vivem com até 1 salário mínimo $(36,4 \%)$.

Com relação aos hábitos de vida, a maioria são não fumantes $(77,3 \%)$, sendo que destes o maior número corresponde às mães (83,3\%). Apenas genitores referiram ser fumantes $(4,6 \%$ do total). Não houve relato de ex-fumantes. Foi observado o mesmo padrão para o consumo de bebida alcoólica: a maioria dos genitores são consomem bebida alcoólica $(64,4 \%)$, destes o maior número corresponde às mães $(74,2 \%)$. Dos etilistas, o maior número é representado pelos pais $(27,3 \%)$. Não houve relato de ex-etilistas.

Dos diagnósticos de câncer em ambos os sexos, o mais prevalente foi a Leucemia Linfoide Aguda (LLA) (34,84\%). A LLA teve maior incidência em indivíduos do sexo masculino, aproximadamente $41,8 \%$, contra $28,6 \%$ no sexo feminino. Não houve similaridade quanto à apresentação dos demais diagnósticos (Gráficos 1 e 2).
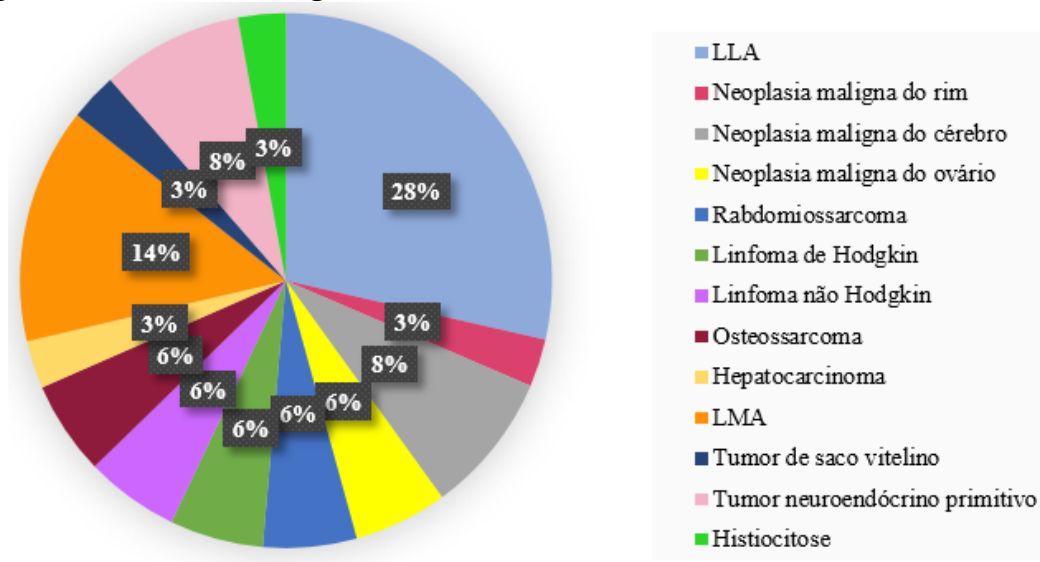

Gráfico 1: Tipos de câncer em crianças do sexo feminino
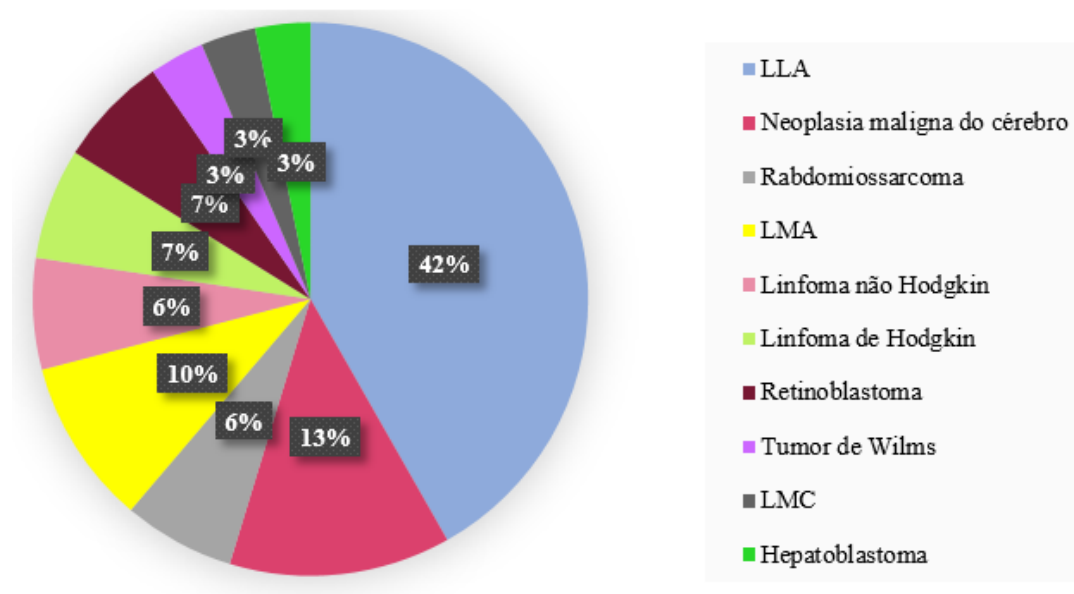

Gráfico 2: Tipos de câncer em pacientes do sexo masculino

Os quimioterápicos mais utilizados foram: vincristina, metrotexato, etoposido, ciclofosfamida e citarabina (mais utilizada: $59,1 \%$ dos casos). Os medicamentos mais utilizados associados ao tratamento de base são: ondansetrona, antibioticoterapia feita com sulfametoxazol e trimetropima, dipirona, dexametasona e omeprazol.

Uma das principais complicações decorrentes do tratamento é o aparecimento de lesões orais, como afta $(7,6 \%)$, candidíase $(6,1 \%)$, herpes $(9,1 \%)$ e gengivite $(1,5 \%)$. A localização das lesões são similares para ambos os sexos, sendo os lábios (29\% no sexo feminino e $26 \%$ no sexo masculino) e a língua ( $22 \%$ no sexo feminino e $26 \%$ no sexo masculino) as regiões mais afetadas. Pode ser classificada em quatro categorias clínicas: graus 
I, II, III, IV. As crianças com maiores complicações, ou seja, aquelas que apresentaram graus III e IV, são do sexo feminino, cerca de $42,9 \%$ para cada grau.

Para o tratamento da mucosite e das demais lesões orais, podem ser usados: laserterapia e laser preventivo, nistatina, fluconazol e oncilon. O laser foi a terapia mais utilizada, chegando a $100 \%$ nas crinaças de sexo feminino, entretanto, apenas crianças do sexo masculino $(44,4 \%)$ fizeram laser preventivo.

\section{DISCUSSÃO}

A caracterização sociodemográfica é de extrema relevância, pois, com a análise das discrepâncias em saúde, é possível reconhecer causas das doenças e identificar meios mais eficientes para preveni-las (Kaplan, 1998). A maioria das crianças apresenta renda familiar de até um salário mínimo e possuem genitores com escolaridade de nível baixo a médio. Isso mostra que grupos populacionais com níveis socioeconômicos mais baixos e baixo nível educacional apresentam maior carga de doença devido à maior incidência de câncer em geral, pelo diagnóstico tardio e dificuldade de acesso ao tratamento adequado (Filho, 2008). A literatura mostra que para esses segmentos da população, o impacto da doença é ainda maior e mais grave, pois já apresentam dificuldades de acesso a bens e serviços para a satisfação de suas necessidades básicas (Carvalho, 2008). Entretanto, a maioria dos genitores não fumam nem consomem bebida alcoólica, o que é fator protetor para a situação de saúde da família.

A maioria dos quimioterápicos acaba lesando as células normais, principalmente aquelas de crescimento rápido, por não agir de forma específica. Assim, altera a microbiota bucal, o volume e a composição salivar e a maturação epitelial, resultando em lesões orais, como a mucosite (Sasada et al, 2013). A toxicidade do quimioterápico depende do mecanismo de ação, dose e interação com outras drogas e os mais tóxicos são: metotrexato, ciclofosfamida, citarabina e ifosfamida, sendo os três primeiros os mais observados no estudo (Curra, 2016). Essa toxicidade e a imunossupressão predispõem à ocorrência de lesões orais (aftas, herpes, candidíase e gengivite), como foi observado nas crianças da presente pesquisa. As infecções mais comuns são: fúngicas, causada por Candida (tem como principal fator de risco a neutropenia grave e persistente) e viral, que tem como agente etiológico o herpes-vírus humano (em pacientes imunocomprometidos, geram lesões mais dolorosas, extensas e de cura mais demorada) (Luiz et al, 2008). A gengivite, hiperplasia gengival e hemorragia são decorrentes do acometimento hematológico e da alteração na distribuição de leucócitos (Kroetz \& Czlusniak, 2003), entretanto apenas uma criança acompanhada no estudo apresentou gengivite.

No entanto, a lesão oral mais frequente e de maior gravidade é a mucosite, o que foi contatado na pesquisa. Ela pode ser definida como uma alteração da mucosa que reveste a cavidade oral (Neville et al, 2009; Sasada et al, 2013). Manifesta-se primeiramente como uma região de coloração esbranquiçada, seguida por edema, eritema e ser friável. Depois, há formação de uma membrana superficial composta por fibrina e pus, amarelada e removível, formando úlceras que podem ser acompanhadas de dor e ardência (Neville et al, 2009). Esse quadro pode levar à modificação ou interrupção do protocolo quimioterápico empregado, além de prejudicar a alimentação, higiene bucal e a comunicação paciente (Sonis, 2004).

Para avaliação clínica da mucosite nos pacientes do estudo, utilizou-se a graduação proposta pela Organização Mundial de Saúde (OMS) (Curra, 2016). Há cinco categorias: grau 0 (sem alterações), grau 1 (dor, inflamação e eritema), grau 2 (eritema e ulceração), grau 3 (ulceração e dieta exclusivamente líquida) e grau 4 (ulceração e o paciente não consegue se alimentar pela boca).

O tratamento da mucosite é feito com o anestésico tópicos, anti-inflamatórios e drogas antimicrobianas. Além disso, atualmente a fotobiomodulação (FBM), também conhecida como laser de baixa intensidade ou de baixa potência, tem se mostrado eficiente na prevenção 
e no reparo das lesões, pois proporciona a cicatrização e a diminuição do processo inflamatório (Curra, 2016), sendo não invasivo e de fácil execução. Foi feito nos pacientes do estudo e apresentou boa aceitação e solucionou mais rapidamente a mucosite.

\section{CONSIDERAÇÕES FINAIS (ou Conclusão)}

Neoplasias em crianças merece atenção especial, pois é grande o desgaste psíquico, físico e social, assim como o custo financeiro. Portanto, é imprescindível o diagnóstico e o tratamento adequado precocemente. No entanto, isso não acontece com frequência para as populações com baixo nível socioeconômico que acompanhamos na pesquisa. Observamos que o tratamento empregado contra as neoplasias gerou diversas complicações, como as lesões orais, sendo mais frequentes nas leucemias, devido à maior toxicidade dos quimioterápicos utilizados nesses casos. A lesão mais frequente e de maior gravidade é a mucosite, pois dificulta a alimentação, higiene bucal e comunicação. No entanto, a terapia com laser de baixa intensidade, associada a medicações paliativas, proporcionou uma recuperação mais rápida. É importante, portanto, a presença de uma equipe multidisciplinar para a prevenção de complicações locais e sistêmicas, bem como para o manejo adequado das lesões orais decorrentes do tratamento.

\section{REFERÊNCIAS}

CARVALHO, C.S.U. 2008. A necessária atenção à família do paciente oncológico. Revista Brasileira de Cancerologia, 54(1): 87-96

CUNHA, C.B. 2010. Avaliação da eficácia do tratamento para mucosite oral induzida por cinco-fluoracil, com uso de laser de baixa potência em diferentes comprimentos de onda. Universidade de São Paulo, Tese.

DINIZ, A. B. et al. 2005. Perfil epidemiológico do câncer infantil em população atendida por uma unidade de oncologia pediátrica em Salvador-Bahia. R. Ci. méd. biol., Salvador 4 (2): 131-139.

FILHO, V.W.; ANTUNES, J.L.F.; BOING, A.F.; LORENZI, R.L. 2008. Perspectivas da Investigação sobre Determinantes Sociais em Câncer. Physis Revista de Saúde Coletiva, Rio de Janeiro, 18 (3): 427-450.

INCA , Instituto Nacional de Câncer José Alencar Gomes da Silva. 2016 [online]. Tipos de câncer: infantil. Homepage: http://www2.inca.gov.br/wps/wcm/connect/tiposdecancer/site/home/infantil.

KAPLAN, G.A. 1998. The role of epidemiologists in eradicability of poverty. Lancet 352: $1627-1628$.

KROETZ, F.M.; CZLUSNIAK; G.D. 2003. Alterações bucais e condutas terapêuticas em pacientes infanto-juvenis submetidos a tratamentos anti-neoplásicos. Publ. UEPG Ci. Biol. Saúde, Ponta Grossa, 9 (2): 41-48.

LUIZ, A.C.; EDUARDO, F.P.; BEZINELLI, L.M.; CORREA, L. 2008. Alterações bucais e cuidados orais no paciente transplantado de medula óssea. Rev. Bras. Hematol. Hemoter. 30 (6):480-487.

MARTINS, A.C.M.; CAÇADOR, N.P.; GAETI, W.P. 2002. Complicações bucais da quimioterapia antineoplásica. Acta Scientiarum 24(3):663-70.

NEVILLE, B.W.; DAMM, D.D.; ALLEN, C.M.; BOUQUOT, J.E. 2009. Patologia oral e maxilofacial. 3. ed. Rio de Janeiro: Guanabara Koogan.

SASADA, I.N.V.; MUNERATO, M.C.; GREGIANIN, L.J. 2013. Mucosite oral em crianças com câncer - revisão de literatura. Rev. Faculdade Odontologia, Passo Fundo 18 (3): 345350 .

SONIS, S.T. 2004. Pathobiology of mucositis. Semin Oncol Nurs 20: 11-15. 